\title{
Cryopreservation of Chlorella vulgaris Using Different Cryoprotectant Agents
}

\author{
Helder Rodrigues da Silva ${ }^{1}$, Francino Costa Palhares da Silva ${ }^{1}$, Cassio Egidio Cavenaghi Prete ${ }^{2}$, \\ Rodrigo Thibes Hoshino ${ }^{2}$, Ricardo Tadeu de Faria ${ }^{2}$, Mario Sergio Mantovani ${ }^{3}$ \& Carmen Luisa Barbosa Guedes ${ }^{1}$ \\ ${ }^{1}$ Postgraduate Program in Bioenergy, Center for Exact Sciences, Universidade Estadual de Londrina, Londrina, \\ Paraná, Brazil \\ ${ }^{2}$ Center for Agricultural Sciences, Department of Agricultural Sciences, Universidade Estadual de Londrina, \\ Londrina, Paraná, Brazil \\ ${ }^{3}$ Center for Biological Sciences, Department of General Biology, Universidade Estadual de Londrina, Londrina, \\ Paraná, Brazil \\ Correspondence: Helder Rodrigues da Silva, Postgraduate Program in Bioenergy, Center for Exact Sciences, \\ Universidade Estadual de Londrina, Paraná, Brazil. Tel: 55-433-371-5453. E-mail: heldersilva@uel.br
}

$\begin{array}{lc}\text { Received: March 1, } 2020 & \text { Accepted: April 7, } 2020 \quad \text { Online Published: June 15, } 2020 \\ \text { doi:10.5539/jas.v12n7p75 } & \text { URL: https://doi.org/10.5539/jas.v12n7p75 }\end{array}$

The research is financed by Coordenação de Aperfeiçoamento de Pessoal de Nível Superior (CAPES-Brazil).

\begin{abstract}
The objective of this study is to evaluate the cryopreservation of Chlorella vulgaris using different substances. The $C$. vulgaris was cultured in medium $\mathrm{MH}$, the microalgae were grown under a 12:12 $\mathrm{h}$ light: dark photoperiod, illumination with $40 \mathrm{~W}$ led lamps, and a controlled temperature of $28 \pm 1{ }^{\circ} \mathrm{C}$. C.vulgaris was cultured for 15 days and the culture was aliquoted into $3-\mathrm{mL}$ cryogenic tubes. The 3-mL aliquot was centrifuged, the supernatant was discarded, and the pellet was resuspended in different cryoprotectant solutions, T1-PVS1, T2-PVS2, T3-PVS2 (1\% phloroglucinol), T4 (2 M glycerol), and T5 (5\% methanol). The samples were rapidly frozen in liquid nitrogen $\left(-196{ }^{\circ} \mathrm{C}\right)$ and analyzed after 15,150 , and 300 days of freezing. Cell viability was determined in cultures grown for 20 days. The only effective treatment was T5, which promoted the growth of thawed cultures in both solid and liquid media. After 15 days of freezing in liquid nitrogen and 20 days of culture growth, the number of viable and nonviable cells was $3.42 \pm 0.72 \times 10^{7}$ and $0.06 \pm 0.009 \times 10^{7}$, respectively, and viability was $98.2 \%$. Similar values were obtained after 150 and 300 days of freezing: $2.17 \pm 0.15 \times 10^{7}$ and $2.35 \pm 0.18 \times 10^{7}$ viable cells, $0.05 \pm 0.02 \times 10^{7}$ and $0.10 \pm 0.02 \times 10^{7}$ nonviable cells, and viability of $97.6 \%$ and $95.8 \%$, respectively. The cryopreservation protocol for microalgae $C$. vulgaris using 5\% methanol was effective; therefore, it is possible to maintain this strain under axenic conditions in liquid nitrogen for long periods.
\end{abstract}

Keywords: liquid nitrogen, conservation, microorganism, microalgae, biotechnology, bioenergy, biofuels

\section{Introduction}

Microalgae are photosynthetic microorganisms that can produce different metabolites, including lipids, proteins, carbohydrates, and pigments. A study has demonstrated the potential of microalgae as a raw material for producing biofuels, nutraceuticals, cosmetics and pharmaceuticals, bioestmulants and biofertilizer for agricultural and for other applications (Andrade et al., 2014; Richmond, 2004; Silva et al., 2016).

One of the challenges in microalgae production is the maintenance of strains in the laboratory. The methodology most commonly used for growing microalgae is subculturing samples isolated in solid or liquid medium at low temperatures and low-light conditions to minimize biological activity and growth (Lorenz, Friedl, \& Day, 2005). This procedure requires the long-term and intensive use of labor and materials and presents the risk of contamination and changes in genetic stability because phenotypic variations occur after successive subculturing over time (Day et al., 2005).

Several studies developed new techniques to preserve these microorganisms and reduce cost and labor, with particular emphasis on the development of effective cryopreservation techniques (Lourenço, 2006). 
Cryopreservation is a methodology used to preserve microorganisms at ultra-low temperatures (less than $-130{ }^{\circ} \mathrm{C}$ ) and allows their growth after thawing (Day \& Brand, 2005; Tessarolli, Day, \& Vieira, 2017).

The advantages of cryopreservation are long-term stability, reduced material costs, protection against genetic drift and contamination, and reduced cost of long-term maintenance (Fernandes et al., 2019; Prakash, Nimonkar, \& Shouche, 2013; Tessarolli et al., 2017). Although the procedures for cryopreserving microorganisms are usually simple, complex cell culture protocols involving multi-stage freezing at specific cooling rates and storage in liquid nitrogen are required for microalgae (Day \& Harding, 2008).

Another determinant factor in the success of cryopreservation is the composition of the freezing medium, in which reagents and concentrations depend on the sensitivity of each strain (Hubalek, 2003). In view of the heterogeneity of microalgae, many strains require specific protocols and standards, and many species do not adapt to the freezing technique (Taylor \& Fletcher, 1998).

A plant vitrification solution (PVS) is used for cryopreserving plant tissues, including embryos, protocorms, pollen, seeds, and cell suspensions (Sakai \& Engelmann, 2007). Nonetheless, no studies have evaluated the potential use of PVS for microalgae.

Given the complexity of the protocols and the need for specific equipment, the objective of this study is to evaluate the effectiveness of different cryoprotective agents in preserving microalgae Chlorella vulgaris.

\section{Materials and Methods}

\subsection{Microalgae and Culture Conditions}

The experiments were carried out in the Laboratory of Chemistry of Biomass, Biofuels, and Bioenergy (Laboratório de Química da Biomassa, Biocombustíveis e Bioenergia-LAQUIBIO) and in the Laboratory of Crop Science of the Universidade Estadual de Londrina-UEL, Londrina, Paraná, Brazil.

A strain of the microalga Chlorella vulgaris was obtained from the Laboratory of Crop Science of UEL and was maintained in solid and liquid culture media under axenic conditions. Subculture in liquid medium under aseptic conditions was performed to obtain the stock culture. MH medium with the following composition was used: $0.57 \mathrm{~g} \mathrm{~L}^{-1} \mathrm{MAP}, 0.075 \mathrm{~g} \mathrm{~L}^{-1} \mathrm{CaCl}_{2}, 0.36 \mathrm{~g} \mathrm{~L}^{-1} \mathrm{KNO}_{3}, 0.225 \mathrm{~g} \mathrm{~L}^{-1} \mathrm{MgSO}_{4}, 0.09 \mathrm{~g} \mathrm{~L}^{-1}$ YaraVita Rexolin BRA® $\left(11.6 \% \mathrm{~K}_{2} \mathrm{O}, 1.28 \% \mathrm{~S}, 0.86 \% \mathrm{Mg}, 2.1 \% \mathrm{~B}, 0.36 \% \mathrm{Cu}, 2.66 \% \mathrm{Fe}, 2.48 \% \mathrm{Mn}, 0.036 \% \mathrm{Mo}\right.$, and $3.38 \% \mathrm{Zn}$ ) (Silva, 2016). The $\mathrm{pH}$ was adjusted to 7.0 using $1 \mathrm{M} \mathrm{KOH}$.

The stock culture was maintained in a growth chamber for 15 days in 1-L Erlenmeyer flasks containing $500 \mathrm{~mL}$ of $\mathrm{MH}$ medium under a 12:12 h light: dark photoperiod, illumination with $40 \mathrm{~W}$ led lamps, and controlled temperature of $28 \pm 1{ }^{\circ} \mathrm{C}$.

\subsection{Cryopreservation Protocols}

After initial culturing, the stock was aliquoted into 3-mL cryogenic tubes. A 3-mL aliquot was centrifuged at $10,000 \mathrm{rpm}$ for $10 \mathrm{~min}$, the supernatant was discarded, and the pellet was resuspended in different freezing media.

The solutions used were (1) PVS1 (19\% glycerol, 13\% ethylene glycol, 13\% propylene glycol, 6\% dimethyl sulfoxide [DMSO], $0.5 \mathrm{M}$ sorbitol); (2) PVS2 (30\% glycerol, 15\% ethylene glycol, 15\% DMSO, $0.4 \mathrm{M}$ sucrose); (3) PVS2 + 1\% phloroglucinol; (4) $2 \mathrm{M}$ glycerol; and (5) 5\% methanol. MH medium frozen in liquid nitrogen without cryoprotectants was used as a control.

The pellets were resuspended in $1.4 \mathrm{~mL}$ of the freezing medium at $4{ }^{\circ} \mathrm{C}$, kept in an ice bath for $20 \mathrm{~min}$, and subjected to rapid freezing in liquid nitrogen $\left(-196^{\circ} \mathrm{C}\right)$. Centrifugations and the addition of the cryoprotective solutions were performed in a laminar flow chamber under aseptic conditions. All assays were performed in triplicate.

\subsection{Thawing and Recovery Growth}

Thawing was performed after 15, 150, and 300 days of freezing. The tubes were removed from the liquid nitrogen and thawed by manual stirring at room temperature.

Test tubes containing $2 \mathrm{~mL}$ of sterile MH medium were inoculated with $200 \mu \mathrm{L}$ of the thawed suspension, without performing centrifugation and washing procedures for removing the cryoprotectants.

In the first thawing time point (15 days), $100 \mu \mathrm{L}$ of the thawed suspension was dispersed in solid MH medium (1\% agar) using a Drigalski loop to assess growth on semisolid medium. At 150 days of freezing, Erlenmeyer flasks containing $100 \mathrm{~mL}$ of $\mathrm{MH}$ medium were inoculated with $800 \mu \mathrm{L}$ of the thawed suspension. 
The inoculated tubes, plates, and Erlenmeyer flasks were kept in a growth chamber for 20 days under the same conditions described for the initial culture. All inoculation procedures were performed in triplicate $(n=3)$ under aseptic conditions.

\subsection{Determination of Cell Viability}

After recovery growth, cell viability was determined by Trypan blue staining. An aliquot of cells was mixed with $0.4 \%$ Trypan blue $($ Life $\AA$ ) dye at a ratio of $1: 1$, and cell viability was quantified in a Neubauer chamber. The percentage of nonviable cells was calculated by the equation: nonviable cells $(\%)=(\mathrm{nI} \times 100) / \mathrm{N}$, where $\mathrm{nI}$ is the number of stained (nonviable) cells and $\mathrm{N}$ is the total number of cells. The optical photomicrography was realized in Laboratory of Electron Microscopy and Microanalysis- UEL, using an optical microscope (Eclipse E200, Nikon) with a 40X objective and a visual magnification of 400X. After determination of the number of cells was realized the mean of 3 replicates $(n=3)$ of recovery growth and calculate the standard deviations (SD), cells viability was measured in $\%$.

\section{Results and Discussion}

The cell count of the stock culture of Chlorella vulgaris was $8.52 \times 10^{6}$ cells $\mathrm{mL}^{-1}$, and the optical density at 670 nm was 0.42 .

The only effective treatment was T5 (5\% methanol) according to the conditions studied, which promoted the growth of the thawed culture in both solid and liquid media after 15, 150, and 300 days of freezing (Figures 1 and 2). In the other treatments, it was not possible to assess the presence of cells in the recovery cultures. Therefore, only the results using $5 \%$ methanol are presented.

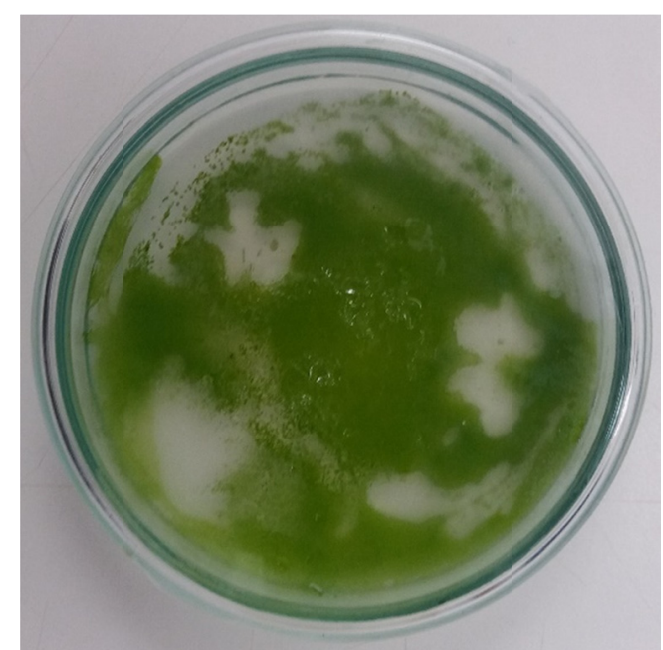

(A)

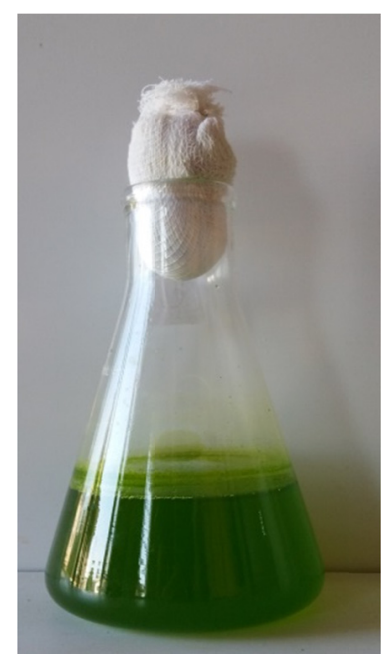

(B)

Figure 1. Cultivation of C. vulgaris in Petri dishes in semisolid MH medium after 15 days of freezing in liquid nitrogen using 5\% methanol (a) and in a liquid medium after 150 days of freezing in liquid nitrogen using $5 \%$ methanol (b)

After 20 days of culture growth, the number of viable and nonviable cells was $3.42 \pm 0.72 \times 10^{7}$ and $0.06 \pm 0.009 \times$ $10^{7}$, respectively, after 15 days of freezing in liquid nitrogen, and cell viability was $98.2 \%$. These values were similar to those at 150 and 300 days of freezing: $2.17 \pm 0.15 \times 10^{7}$ and $2.35 \pm 0.18 \times 10^{7}$ viable cells, $0.05 \pm 0.02 \times$ $10^{7}$ and $0.10 \pm 0.02 \times 10^{7}$ nonviable cells, and cell viability of $97.6 \%$ and $95.8 \%$, respectively (Figure 2 ). The results of the viability assay indicated the presence of viable (unstained) and nonviable (stained) cells in T5 (Figure 3). 


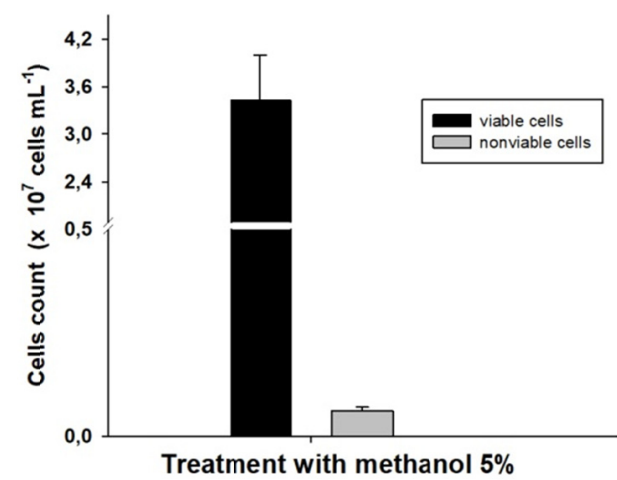

A

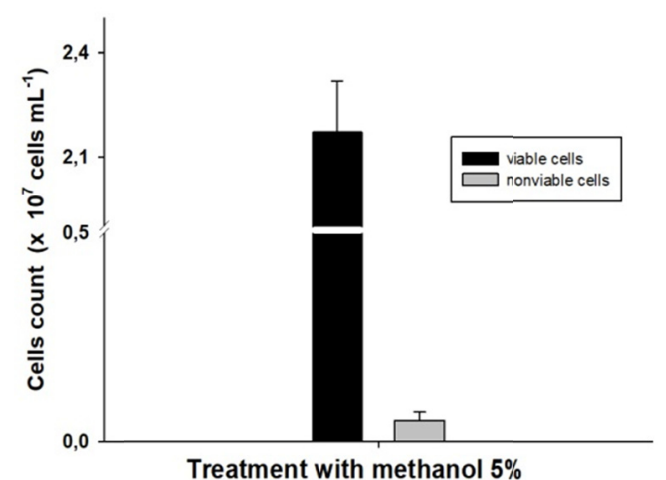

B

300 days

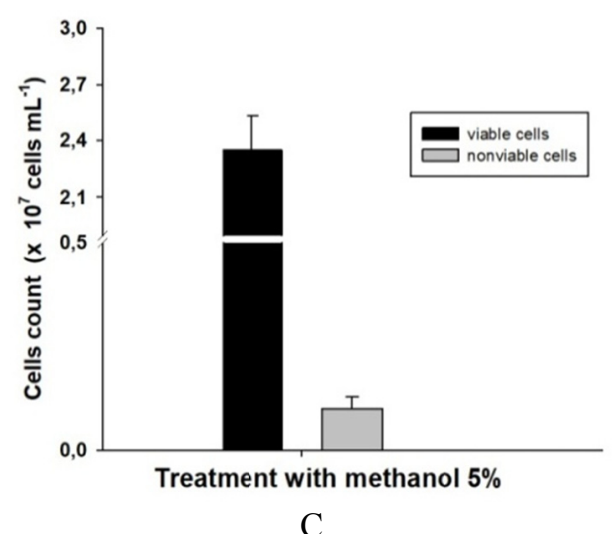

Figure 2. Count of viable and nonviable cells after 20 days of culture of Chlorella vulgaris cryopreserved in liquid nitrogen for 15(A), 150 (B), and 300 (C) days using 5\% methanol. Data shown is the mean $\pm \mathrm{SD}, \mathrm{n}=3$

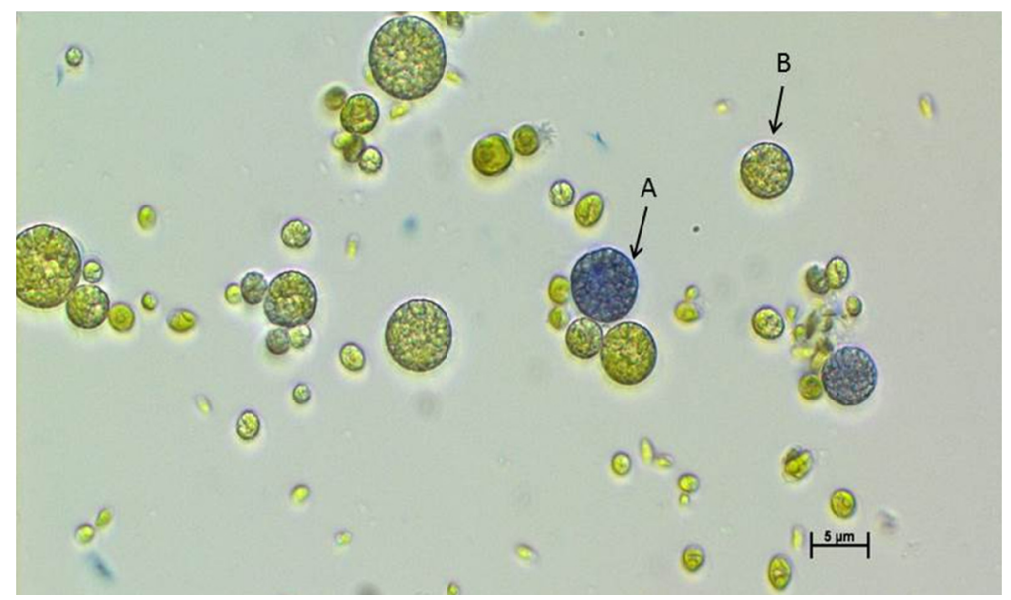

Figure 3. Optical photomicrography of Chlorella vulgaris cells using Trypan blue after different freezing periods. (A) stained nonviable cells; (B) unstained viable cells

This assay quantifies cell viability by measuring the percentage of cells with permeabilized plasma membranes. The membranes of dead cells and cells damaged by freezing are ruptured and allow the dye to enter the 
cytoplasm. The percentage of viable cells is analyzed by microscopy (Fang \& Trewyn, 2012). This methodology has been used to distinguish between living and dead cells in different microalgae species (Huang, Lin, Pan, Huang, \& Chu, 2015; Sánchez Mirón, Cerón García, García Camacho, Molina Grima, \& Chisti, 2002; Song, Kang, Jeong, Kim, \& Lim, 2018).

Several factors may affect the efficiency of cryopreservation protocols, including cell density of stock cultures, number of centrifugation steps, light intensity during culturing, composition of the freezing medium, and the interactions of cryoprotectants with different species of microorganisms (Canavate \& Lubián, 1995).

In the scientific literature the cryoprotective potential of several substances was evaluated. The most effective substances were DMSO, methanol, ethylene glycol, and propylene glycol, and the least effective were glycerol, polyethylene glycol, PVP, and sucrose. DMSO is widely used as a freezing medium but is toxic to most microorganisms (Hubalek, 2003); therefore, many studies seek to define optimal combinations and concentrations of cryoprotectants.

Bui et al. (2013) demonstrated the synergistic effect of DMSO combined with $200 \mathrm{mM}$ sucrose. The optimal concentration of DMSO was 6.5\%, and higher concentrations reduced cell viability in Palmellopsis sp. and Chlamydomonas sp. For Chlorella vulgaris, Nannochloropsis oculata, and Tetraselmis tetrathele, 2.5\% or 5.0\% DMSO in isolation has no cryoprotective effect. However, the combination of 5\% DMSO and 5\% ethylene glycol resulted in survival of $10 \%$ for Chlorella vulgaris, and survival increased to approximately 50\% when 5\% proline was added to the freezing medium (Nakanishi, Deuchi, \& Kuwano, 2012).

The viability of Chlorella sorokiniana using 7\% DMSO was 80\%. Nonetheless, for Desmodesmus spinosus and Chlamydomonas biconvexa, the combination of 5\% glycerol and 5\% PEG-400 resulted in viability higher than $48 \%$ and $70 \%$, respectively, at $24 \mathrm{~h}$ after thawing (Fernandes et al., 2019).

Although the DMSO concentration in PVS1 (6\%) was below the lethal concentration described in the literature, treatment with DMSO was ineffective. GWO et al. (Gwo, Chiu, Chou, \& Cheng, 2005) showed that the toxicity threshold of ethylene glycol, DMSO, methanol, and polyethylene glycol as cryoprotectants for Nannochloropsis oculata was 20\%,30\%,30\%, and 40\%, respectively. However, Joseph, Panigrahi, and Chandra (2000) observed that these limits varied according to species and, for Chaetoceros calcitrans and Chlorella marina, DMSO or methanol concentrations higher than $10 \%$ were lethal during a 15-min exposure, which could justify the ineffectiveness of treatments using PVS2 (30\% glycerol, 15\% ethylene glycol, 15\% DMSO, $0.4 \mathrm{M}$ sucrose).

Another critical factor to consider is the cooling rate. The above studies used slow cooling in two stages, in which, after adding the cryoprotectants, the tubes were transferred to freezers and frozen at a cooling rate at $-1{ }^{\circ} \mathrm{C} \mathrm{min}^{-1}$ until they reached temperatures of $-40{ }^{\circ} \mathrm{C}$ to $-80{ }^{\circ} \mathrm{C}$. The tubes remained at this temperature range for 4 to $16 \mathrm{~h}$ and were then immersed in liquid nitrogen.

Buhmann, Day, and Kroth (2013) found that the cryopreservation of Planothidium frequentissimum using 5\% DMSO was feasible by pre-freezing at $-40{ }^{\circ} \mathrm{C}$ at a controlled cooling rate of $1{ }^{\circ} \mathrm{C} \mathrm{min}-1$ and maintenance in the dark for 48 hours in the post-thawing recovery phase to reduce photooxidative stress. Morschett, Reich, Wiechert, and Oldiges (2016) reported that direct freezing in liquid nitrogen reduced the viability of Chlorella vulgaris maintained in 10\% DMSO, 10\% ethylene glycol, and 10\% L-proline.

Another factor that may have affected the effectiveness of PVS is the step of washing and removing the cryoprotective agents. The steps performed after thawing included centrifuging the tubes, discarding the supernatants, and resuspending the pellet in culture medium or saline before inoculation in the recovery medium. Although decreasing the number of centrifugations increases cell viability by reducing mechanical damage (Bui et al., 2013), skipping the centrifugation washing step may be detrimental because of the high toxicity of the cryoprotectants at room temperature, even at low concentrations, diluted in the recovery medium.

These results indicate the advantages of T5 because the cryopreservation protocol used both before and after freezing is simple, and some steps are unnecessary, including controlled cooling, culture maintenance at pre-freezing temperatures, centrifugation to remove the cryoprotective agents, and recovery growth under low-light conditions. These characteristics make the T5 protocol simple, fast, and effective for preserving Chlorella vulgaris.

Scarbrough and Wirschell (2016) have shown that 5\% methanol is better than commercial kits for preserving Chlamydomonas reinhardtii because the thawed culture can be directly inoculated in semisolid agar or liquid medium under aeration. Furthermore, aeration was essential to guarantee cell viability during culture recovery in liquid medium. 
The present results demonstrate that Chlorella vulgaris can be inoculated directly in a liquid medium without the need for agitation or aeration. Immediately after manual thawing at room temperature, the sample $(1.0-1.5 \mathrm{~mL})$ can be used to inoculate $100 \mathrm{~mL}$ of liquid medium.

\section{Conclusions}

The cryopreservation of microalgae Chlorella vulgaris was effective, allowing its long-term preservation under axenic laboratory conditions and cell growth in a liquid culture medium, which is used for large-scale biomass production in different biotechnological applications.

\section{Acknowledgements}

The authors are grateful to the Coordenação de Aperfeiçoamento de Pessoal de Nível Superior (CAPES-Brazil) for providing a postdoctoral scholarship (PNPD program) to Dr. Helder Rodrigues da Silva, a master scholarship to Francino Costa Palhares da Silva, and a research productivity scholarship to Professor Ricardo Tadeu de Faria

\section{References}

Andrade, D. S., Filho, A. C., Guedes, C. L. B., Lima, F. A. F., Machineski, G. S., Matos, M. A., \& Silva, H. R. (2014). Principais produtos da biomassa algal e suas aplicações biotecnológicas. In D. S. Andrade \& A. C. Filho (Eds.), Potencialidades e desafios do cultivo (Vol. 1, pp. 265-286). Londrina: IAPAR.

Buhmann, M. T., Day, J. G., \& Kroth, P. G. (2013). Post-cryopreservation viability of the benthic freshwater diatom Planothidium frequentissimum depends on light levels. Cryobiology, 67(1), 23-29. https://doi.org/ 10.1016/j.cryobiol.2013.04.005

Bui, T. V., Ross, I. L., Jakob, G., \& Hankamer, B. (2013). Impact of procedural steps and cryopreservation agents in the cryopreservation of chlorophyte microalgae. PloS ONE, 8(11), e78668. https://doi.org/ 10.1371/journal.pone.0078668

Canavate, J. P., \& Lubián, L. (1995). Some aspects on the cryopreservation of microalgae used as food for marine species. Aquaculture, 136, 277-290. https://doi.org/10.1016/0044-8486(95)01056-4

Day, J., Benson, E., Harding, K., Knowles, B., Idowu, M., Bremner, D., \& Lorenz, M. (2005). Cryopreservation and conservation of microalgae: the development of a pan-European scientific and biotechnological resource (the COBRA project). CryoLetters, 26(4), 231-238.

Day, J., \& Brand, J. (2005). Cryopreservation methods for maintaining microalgal cultures. Algal Culturing Techniques, 165-187. https://doi.org/10.1016/B978-012088426-1/50013-5

Day, J. G., \& Harding, K. (2008). Cryopreservation of algae. Plant cryopreservation: A practical guide (pp. 95-116). Springer. https://doi.org/10.1007/978-0-387-72276-4_6

Fang, I. J., \& Trewyn, B. G. (2012). Chapter 3:Application of Mesoporous Silica Nanoparticles in Intracellular Delivery of Molecules and Proteins. In N. Düzgüneş (Ed.), Methods in Enzymology (Vol. 508, pp. 41-59). Academic Press. https://doi.org/10.1016/B978-0-12-391860-4.00003-3

Fernandes, M. S., Calsing, L. C. G., Nascimento, R. C., Santana, H., Morais, P. B., de Capdeville, G., \& Brasil, B. S. A. F. (2019). Customized cryopreservation protocols for chlorophytes based on cell morphology. Algal Research, 38, 101402. https://doi.org/10.1016/j.algal.2018.101402

Gwo, J.-C., Chiu, J.-Y., Chou, C.-C., \& Cheng, H.-Y. (2005). Cryopreservation of a marine microalga, Nannochloropsis oculata (Eustigmatophyceae). Cryobiology, 50(3), 338-343. https://doi.org/10.1016/ j.cryobiol.2005.02.001

Huang, L.-F., Lin, J.-Y., Pan, K.-Y., Huang, C.-K., \& Chu, Y.-K. (2015). Overexpressing Ferredoxins in Chlamydomonas reinhardtii Increase Starch and Oil Yields and Enhance Electric Power Production in a Photo Microbial Fuel Cell. International Journal of Molecular Sciences, 16(8), 19308-19325. https://doi.org/10.3390/ijms160819308

Hubalek, Z. (2003). Protectants used in the cryopreservation of microorganisms. Cryobiology, 46(3), $205-229$. https://doi.org/10.1016/s0011-2240(03)00046-4

Joseph, I., Panigrahi, A., \& Chandra, P. K. (2000). Tolerance of three marine microalgae to cryoprotectant dimethy sulfoxide, methanol and glycerol. Indian Journal of Marine Sciences, 29(3), 243-247.

Lorenz, M., Friedl, T., \& Day, J. G. (2005). Maintenance of Actively Metabolizing Microalgal Cultures. Algal Culturing Techniques, 145. https://doi.org/10.1016/B978-012088426-1/50011-1

Lourenço, S. O. (2006). Cultivo de microalgas marinhas-princípios e aplicações. Rima. 
Morschett, H., Reich, S., Wiechert, W., \& Oldiges, M. (2016). Simplified cryopreservation of the microalga Chlorella vulgaris integrating a novel concept for cell viability estimation. Engineering in Life Sciences, 16(1), 36-44. https://doi.org/10.1002/elsc.201500056

Nakanishi, K., Deuchi, K., \& Kuwano, K. (2012). Cryopreservation of four valuable strains of microalgae, including viability and characteristics during 15 years of cryostorage. Journal of Applied Phycology, 24(6), 1381-1385. https://doi.org/10.1007/s10811-012-9790-8

Prakash, O., Nimonkar, Y., \& Shouche, Y. S. (2013). Practice and prospects of microbial preservation. FEMS Microbiology Letters, 339(1), 1-9. https://doi.org/10.1111/1574-6968.12034

Richmond, A. (2004). Handbook of microalgal culture: Biotechnology and applied phycology. Blackwell Science Ltd.

Sakai, A., \& Engelmann, F. (2007). Vitrification, encapsulation-vitrification and droplet-vitrification: A review. CryoLetters, 28(3), 151-172.

Sánchez Mirón, A., Cerón García, M.-C., García Camacho, F., Molina Grima, E., \& Chisti, Y. (2002). Growth and biochemical characterization of microalgal biomass produced in bubble column and airlift photobioreactors: Studies in fed-batch culture. Enzyme and Microbial Technology, 31(7), 1015-1023. https://doi.org/10.1016/S0141-0229(02)00229-6

Scarbrough, C., \& Wirschell, M. (2016). Comparative analysis of cryopreservation methods in Chlamydomonas reinhardtii. Cryobiology, 73(2), 291-295. https://doi.org/10.1016/j.cryobiol.2016.07.011

Silva, H. R. (2016). Produção de microalga Neochloris oleoabundans em diferentes sistemas de cultivo. Universidade Estadual de Londrina, Brazil.

Silva, H. R., Prete, C. E. C., Zambrano, F., Mello, V. H., Tischer, C. A., \& Andrade, D. S. (2016). Combining glucose and sodium acetate improves the growth of Neochloris oleoabundans under mixotrophic conditions. AMB Express, 6(1), 1-11. https://doi.org/10.1186/s13568-016-0180-5

Song, Y. S., Kang, C., Jeong, J., Kim, K.-O., \& Lim, E. (2018). Rheological Analysis of Live and Dead Microalgae Suspensions. Journal of the Korean Physical Society, 72(8), 858-862. https://doi.org/10.3938/ jkps. 72.858

Taylor, R., \& Fletcher, R. L. (1998). Cryopreservation of eukaryotic algae-A review of methodologies. Journal of Applied Phycology, 10(5), 481-501. https://doi.org/10.1023/A:1008094622412

Tessarolli, L. P., Day, J. G., \& Vieira, A. A. H. (2017). Establishment of a cryopreserved biobank for the Culture Collection of Freshwater Microalgae (CCMA-UFSCar), São Paulo, Brazil. Biota Neotropica, 17(2). https://doi.org/10.1590/1676-0611-bn-2016-0299

\section{Copyrights}

Copyright for this article is retained by the author(s), with first publication rights granted to the journal.

This is an open-access article distributed under the terms and conditions of the Creative Commons Attribution license (http://creativecommons.org/licenses/by/4.0/). 